\title{
Zukunftsvisionen für die Gesundheitspolitik aus dermatologischer Sicht!
}

\author{
Future Perspectives for Health-Care Politics from the Dermatologist's Point of View!
}

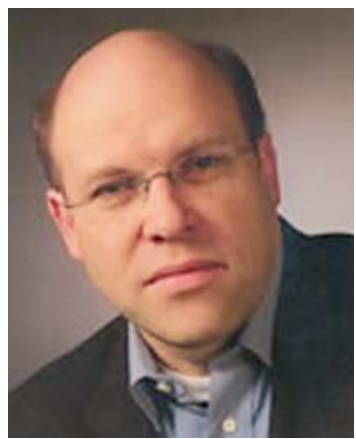

Dr. Matthias Herbst
Kaum ist die Ära Ulla Schmidt vorbei, fragen wir uns, was bleibt, und hoffen, dass vieles besser wird. Zur Beurteilung der Qualität deutscher Gesundheitspolitik eignet sich am besten die Analyse der Auswirkung der Gesetzgebung in Bezug auf das Verhältnis Arzt und Patient. Ein engagierter Arzt ist Voraussetzung für einen zufriedenen Patienten. Ein zufriedener Patient ist auch bereit, für seine Behandlung Geld zu investieren. Nichts ist schlimmer für einen engagierten Arzt, als einen Patienten zu behandeln, der glaubt, dass er Anspruch auf alles hat und alles nichts kostet. Aus diesem Anspruchsdenken heraus entsteht eine vollkommen ungesunde und unwirtschaftliche Erwartungshaltung des Bürgers in Bezug auf die Leistungsfähigkeit der Gemeinschaft, sprich des Staates. Aber auch ein leistungsfernes staatsnahes Gesundheitssystem erweist sich als Problem, insbesondere, wenn Ideologien statt Pragmatismus darin Platz greifen:

Wir Dermatologen erleben es täglich: Ein willkürliches Regelleistungsvolumen (Beispiel Hessen) von 13,80 Euro pro Quartal pro Patient, das in vielen Bundesländern unterhalb der vom System in Euro garantierten Grund-Pauschale liegt (14,50 Euro), ist spätestens seit Einführung des EuroZeitalters in der Abrechnung ein für jeden Bürger nachvollziehbarer Widerspruch in sich. Eine qualifizierte allergologische Diagnostik und Therapie, die wiederum durch dieses Regelleistungsvolumen von 13,80 Euro pro Quartal brutto abgebildet wird und die alle Testungen, Besprechungen und therapeutischen Bemühungen, wie z.B. die etlichen subkutanten Hypersensibilisierungen, umfasst, ist ebenfalls für dieses Regelleistungsvolumen nicht vorstellbar, aber in vielen Praxen entgegen aller betriebswirtschaftlicher Vernunft noch (!) Realität.

Wenn dann der frustrierte Dermatologe und Allergologe erfährt, dass für z.T. dieselbe Leistung im Hausarztbereich mehr als das 4-Fache vergütet wird, muss auch der letzte Laie und Politiker merken, dass in diesem System massive Ungerechtigkeiten vorkommen, die durch keine Logik zu rechtfertigen sind und eindeutig dem Gleichheitsgrundsatz des Grundgesetzes widersprechen.
Es bleibt zu hoffen, dass die schwarz-gelbe Koalition aus diesen Fehlern lernt:

1. Ein von Unternehmen und Angestellten paritätisch finanzierter Gesundheitsfond als Basisfinanzierung braucht individuell die Möglichkeit, Leistungen zusätzlich auf eigene Rechnung versichern zu können.

2. Gleiches Geld für gleiche Leistung, mehr Geld für die qualifiziertere Leistung.

3. Einbindung des Patienten in die Abrechnung, sprich den Wert der Leistung durch eine prozentuale Selbstbeteiligung mit Höchstwertbegrenzung.

4. Stärkung der regionalen Kompetenz von Ärzten und Kassen, um eine leistungsfähige und effektive regionale Gesundheitswirtschaft $\mathrm{zu}$ etablieren (Direktion Gesundheit Region X). Stärkung der Kompetenz der kleineren Fächer in Bezug auf die Mitwirkung.

5. Gleichzeitige Unvereinbarkeit von übergreifenden Ärzte-Mandaten in Gremien wie KV und Berufsverband. Stattdessen Professionalisierung (gute Vertretung gegen gutes Geld!).

Hier ließe sich noch viel diskutieren, aber eines ist wichtig, es muss Schluss sein mit der lähmenden Überbürokratisierung eines Gesundheitswesens, das von der Basis, sprich den Anforderungen von Arzt und Patient her, wachsen muss und nicht zum Selbstzweck einer unüberschaubaren Mammut-Bürokratie werden darf. Mit Qualität, Leistungstransparenz und diagnostischer wie therapeutischer Effizienz sollte sich der Kampf um die Ressourcen seitens der Fach- wie der Ärzteschaft in Klinik wie Praxis gewinnen lassen.

Wichtig ist die Ausgestaltung nicht gegen die Hausärzte, sondern durch Abbildung sinnvoller und effizienter regionaler Behandlungspfade mit den Grundversorgern. Was wieder zählen muss, ist die ärztliche Leistung, sei es in der Praxis oder der Klinik. Ein mündiger und zufriedener Patient ist der Maßstab.

Bemerkenswert ist, dass ich in Diskussionsrunden mit Kassenvorständen immer wieder breite Zustimmung zu diesen Forderungen erhalten habe. Die Dermatologen in Südhessen haben ein klares Zukunftskonzept. Vielleicht sind wir, sprich alle Player im Gesundheitswesen, gar nicht so weit weg voneinander entfernt und brauchen nur etwas Mut, wie auch die Politik...
Dol $10.1055 / \mathrm{s}-0029-1215288$

Akt Dermatol 2009; 35; 435

(c) Georg Thieme Verlag KG .

Stuttgart · New York

ISSN 0340-2541

Korrespondenzadresse

Dr. Matthias Herbst

Furtwänglerstraße 5

69121 Heidelberg

dr.med.matthias.herbst@t-online.de 